\title{
Situational analysis: regional review of the substance use Atlas 2015
}

Elise Gehring ${ }^{1}$ and Khalid Saeed ${ }^{2}$

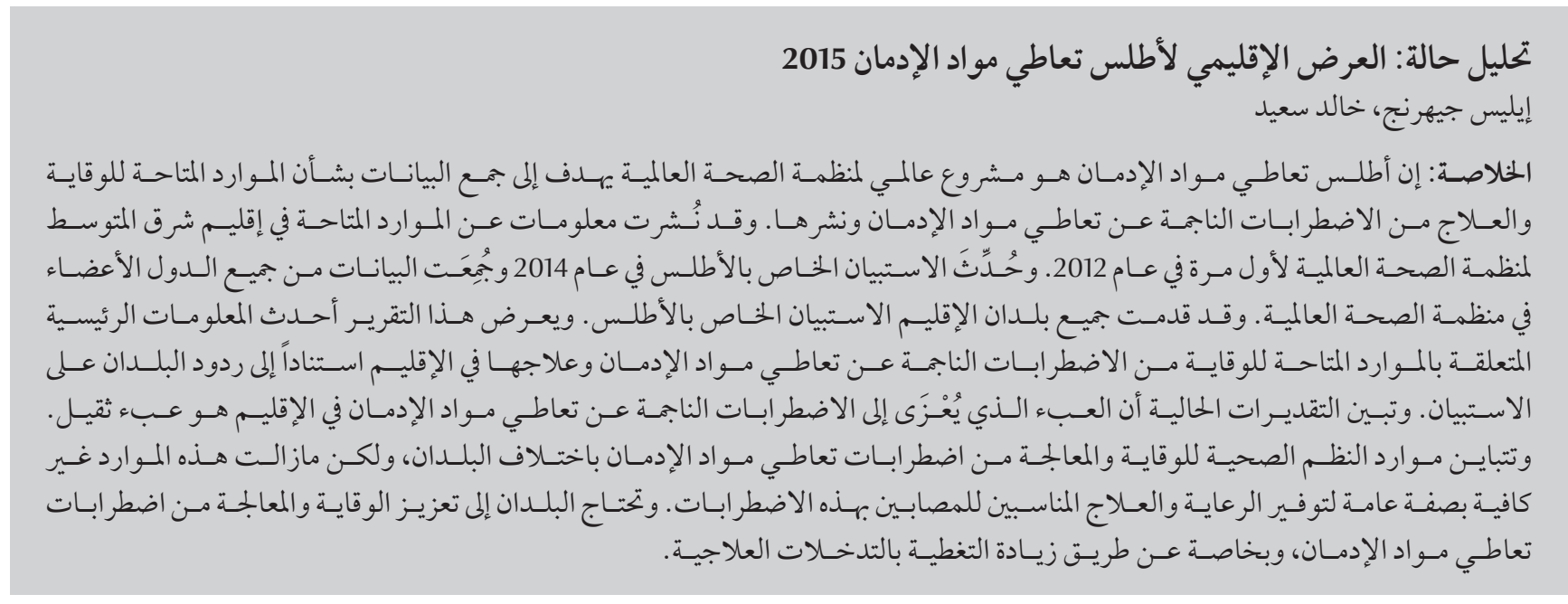

ABSTRACT The Atlas project on substance use is a global WHO project that aims to collect and disseminate data on resources for the prevention and treatment of substance use disorders. Information on resources available in the WHO Eastern Mediterranean Region was first published in 2012. The Atlas questionnaire was updated in 2014 and data were collected in all WHO Member States. All countries of the Region submitted the Atlas questionnaire. This report presents the latest key information on resources for the prevention and treatment of substance use disorders in the Eastern Mediterranean Region based on the responses of the countries to the questionnaire. Current estimates show that the burden attributable to drug use disorders in the Region is high. Health system resources for the prevention and treatment of substance use disorders vary across countries, but are generally still insufficient to provide adequate care and treatment for people with these disorders. Countries need to strengthen prevention and treatment of substance use disorders, particularly by increasing coverage of treatment interventions.

\section{Analyse de situation : examen régional de l'Atlas 2015 sur l'utilisation de substances psychoactives}

RÉSUMÉ Le projet d'Atlas sur l'utilisation de substances psychoactives est un projet mondial de l'OMS visant à collecter et à diffuser des données sur les ressources portant sur la prévention et le traitement des troubles liés à I'utilisation de substances psychoactives. Des informations sur les ressources disponibles dans la Région OMS de la Méditerranée orientale ont été publiées pour la première fois en 2012. Le questionnaire de l'Atlas a été mis à jour en 2014 et des données ont été collectées dans tous les États Membres de l'OMS. Tous les pays de la Région ont soumis le questionnaire de l'atlas. L'article en question présente les informations essentielles les plus récentes sur la prévention et le traitement des troubles liés à l'utilisation de substances psychoactives dans la Région de la Méditerranée orientale sur la base des réponses au questionnaire fournies par les pays. Les estimations actuelles montrent que la charge attribuable aux troubles liés à l'utilisation de substances psychoactives dans la Région est élevée. Les ressources des systèmes de santé pour la prévention et le traitement de ces troubles varient d'un pays à l'autre, mais elles sont généralement insuffisantes pour assurer des soins et des traitements adéquats pour les personnes atteints de ces troubles. Les pays doivent renforcer la prévention et le traitement des troubles liés à I'utilisation de substances psychoactives, notamment en augmentant la couverture des interventions thérapeutiques..

'Department of Mental Health and Substance Abuse, World Health Organization, Geneva, Switzerland (Correspondence to: Elise Gehring: gehringe@ who. int). ${ }^{2}$ Mental Health and Substance Abuse Unit, Department of Noncommunicable Diseases and Mental Health, World Health Organization, Regional Office for the Eastern Mediterranean, Cairo, Egypt.

Received: 19/05/16; accepted: 30/03/17 


\section{Introduction}

The use of alcohol and other psychoactive substances alters brain functions, such as mood, perception and consciousness (1). It can lead to physical and psychological dependence, coercing a person to continue taking the drug despite adverse consequences. People with substance use disorders may suffer from psychological and psychosocial problems, loss of employment or legal problems $(2,3)$. It is a chronic mental disorder characterized by remissions and relapses and frequently accompanied by other mental conditions, such as depression or anxiety disorder, or by physical conditions such as HIV/AIDS, hepatitis $B$ and $C$ and tuberculosis.

Information is limited on substance use in the countries of the Eastern Mediterranean Region of the World Health Organization, which include Afghanistan, Bahrain, Djibouti, Egypt, Islamic Republic of Iran, Iraq, Jordan, Kuwait, Lebanon, Libya, Morocco, Oman, Pakistan, Palestine, Qatar, Saudi Arabia, Somalia, Sudan, Syrian Arab Republic, Tunisia, United Arab Emirates and Yemen.

Alcohol use in the Region is among the lowest worldwide. According to the Global Status Report on Alcohol and Health 2014 (2), the total alcohol per capita consumption in 2010 was $0.7 \mathrm{~L}$, which is almost 9 times lower than the global estimate $(6.2 \mathrm{~L})$. However, more than half of the alcohol consumed in the Region is unrecorded, which means it is not accounted for in official statistics on alcohol taxation or sales.

Information on the prevalence of drug use in the Region is scarce. Data from the World Drug Report 2016 are available for 6 to 13 countries depending on the drug type (3). Cannabis is reported as the most commonly used substance in the Region. The regional median annual prevalence of cannabis use for the population aged 15-64 years is estimated to be $3.6 \%$. Some countries of the Region (Afghanistan,
Islamic Republic of Iran and Pakistan) have among the highest annual prevalence of opioid use in the world, ranging between $2 \%$ and $3 \%$ of the population aged 15-64 (4). This estimate is lower $(<0.5 \%)$ for the other countries of the Region for which data are available.

As mentioned above, drug dependence is one of the consequences of drug use. Based on the findings from the Global Burden of Disease Study 2010 (5), the prevalence of cannabis dependence was estimated to be $0.14 \%$ in the North Africa and Middle East region, which is slightly less than the global average $(0.19 \%)$. Opioid dependence is the most prevalent drug dependence in this region at $0.29 \%$.

Given the complexity of the health and social consequences of substance use disorders, management of substance use disorders involves a range of prevention, treatment and rehabilitation approaches. In addition to specialized services for drug use disorders, a good treatment system should include non-specialized services such as primary health care services as well as social care services (6). A wide range of treatment methods should be available ranging from targeted prevention programmes, screening and brief interventions, to pharmacological treatment and rehabilitation.

Delivery of adequate care and treatment to people with substance use disorders requires a well-functioning prevention and treatment system. Such a health system should have 6 core components: service delivery, health workforce, health information systems, access to essential medicines, financing and leadership/governance (7). These building blocks represent the essential features of a functioning health system.

In 2010, the WHO launched the Atlas project on resources for the prevention and treatment of substance use disorders. The objective of this project is to collect, compile and disseminate information on resources for the prevention and treatment of substance use disorders (8). A first assessment was conducted in 2012 in order to map resources available in the Eastern Mediterranean Region (9). Fourteen countries, representing $92 \%$ of the Region's population, participated in this assessment. Scarcity of available information was one of the main challenges in developing the report. The following key findings were reported. Cannabis was the most prevalent substance used in the Region. Opioids were the main psychoactive substances reported at treatment entry. Even though a drug unit was available in most countries, few countries reported a specific budget for the prevention and treatment of substance use disorders, and out-of-pocket payment was the main financing source for treatment services for substance use disorders. Opioid substitution therapy, which is recommended by WHO as treatment for opioid dependence (10), was reported by $14 \%$ of the countries. Mental health care services are the main providers of treatment for substance use disorders. Of all the WHO regions, the Eastern Mediterranean Region has the lowest number of groups and agencies working in the area of substance use, as well as of screening and brief intervention programmes. With regard to information systems, only $36 \%$ of the countries of the Region had a system for collection of epidemiological data collection about substance use and substance use disorders.

Important United Nations resolutions have been adopted and published over the past 10 years, the most recent being the Special Session of the United Nations General Assembly on the World Drug Problem held in 2016. In 2009, Member States of the United Nations adopted the Political Declaration and Plan of Action on International Cooperation towards an Integrated and Balanced Strategy to Counter the World Drug Problem (11). In 2010, the World Health Assembly endorsed the Global Strategy to Reduce the Harmful 
Use of Alcohol (12). In view of these political changes, the WHO regularly monitors the resources available in an attempt to assess the treatment gap for substance use disorders and what is being done to address this gap. In this regard, the Atlas project on substance use was updated in 2014.

The Sustainable Development Goals that were adopted by leaders worldwide in January 2016 include a specific goal related to substance abuse. Countries around the world are all urged to "strengthen the prevention and treatment of substance abuse, including narcotic drug abuse and harmful use of alcohol". In view of this recent political engagement, information on resources available for the prevention and treatment of substance use disorders is needed. The aim of this review is to present the main findings of the Atlas project update for the Eastern Mediterranean Region. The data presented in this review represent the latest information on resources available in the Region.

The Atlas project refers to specific treatment services for substance use disorders that are defined as follows. Detoxification refers to a relatively short-term treatment aimed at withdrawing an individual from the effects of psychoactive substance use. Treatment refers to the different treatment methods beyond detoxification that are implemented on an outpatient/ambulatory basis (i.e. without formal hospital admission), or on an inpatient basis. Rehabilitation is defined as a longerterm process aimed at enabling people with substance use disorders to achieve an optimal state of health, psychological functioning and social well-being through a combination of approaches. Opioid agonist maintenance therapy refers to the administration of thoroughly evaluated opioid agonist for the management of opioid dependence (10).

The information provided in this review may assist countries to assess their current resources and to compare them with countries with a similar situation as well as to assist in developing evidencebased policies, plans and programmes to effectively address the issues of substance use and related problems.

\section{Methods}

The Atlas project on resources for the prevention and treatment of substance use disorders was launched in 2010 by WHO in order to map resources available for the prevention and treatment of substance use disorders. To update this information, the project was conducted again in 2014 and the questionnaire updated based on the response rate and quality of the initial questionnaire. The final questionnaire contained information on the following areas: policy, legislation and financing, human resources and training, services and interventions, and health information systems. In particular, the list of indicators included governance structure, financing sources, number of beds, availability of training for professionals, availability of medications, treatment coverage for substance dependence, and availability of treatment services for women or children and adolescents.

The Eastern Mediterranean Regional Office collaborated with the responsible ministries in the countries to nominate a focal point for completion of the questionnaire. The focal point was encouraged to consult with experts and gather relevant information to answer the questions. The questionnaire was made available through an online platform as well as in a Word document upon request. Technical support was provided by $\mathrm{WHO}$ during the submission period.

Once a filled questionnaire was received, it was screened for incomplete and inconsistent answers. To ensure high quality data, respondents were contacted again and asked to respond to requests for clarification and to correct their responses. Data were analysed on a regional basis based on the number of countries that provided a response to a specific item.

\section{Results}

All of the 22 countries of the Eastern Mediterranean Region submitted the questionnaire. However, the response rate varied between the questions. Results are presented below by area.

\section{Leadership and governance}

Countries were asked about the presence of a government unit or focal person responsible for a policy regarding substance use prevention and treatment. The majority of countries (18/22, $82 \%$ ) have a government unit responsible for prevention policies, and for the majority this unit is responsible for both alcohol and drug use. The majority of countries also had a government unit responsible for a policy on the treatment of substance use disorders $(18 / 20$, $90 \%$ ). Figure 1 shows the countries' responses regarding the government unit or focal person responsible for prevention of substance use and treatment of substance use disorders. The different response options for prevention were: i) 1 unit responsible for the prevention of both alcohol and drug use, ii) 2 separate units for the prevention of alcohol and drug use, or iii) 1 unit responsible for the prevention of drug use only. Similarly, the different response options for treatment were: i) 1 unit responsible for the treatment of alcohol and drug use disorders or ii) 1 unit responsible for the treatment of drug use disorders only.

In most of the countries the unit or focal person is responsible for policies covering other areas. In 12 of 15 countries (80\%), the unit responsible for treatment policy is also responsible for mental health policy. A few countries $(3 / 15,20 \%)$ reported that this unit is responsible for criminal justice. Prevention is under the same unit as mental health in most of the countries (10/14, 


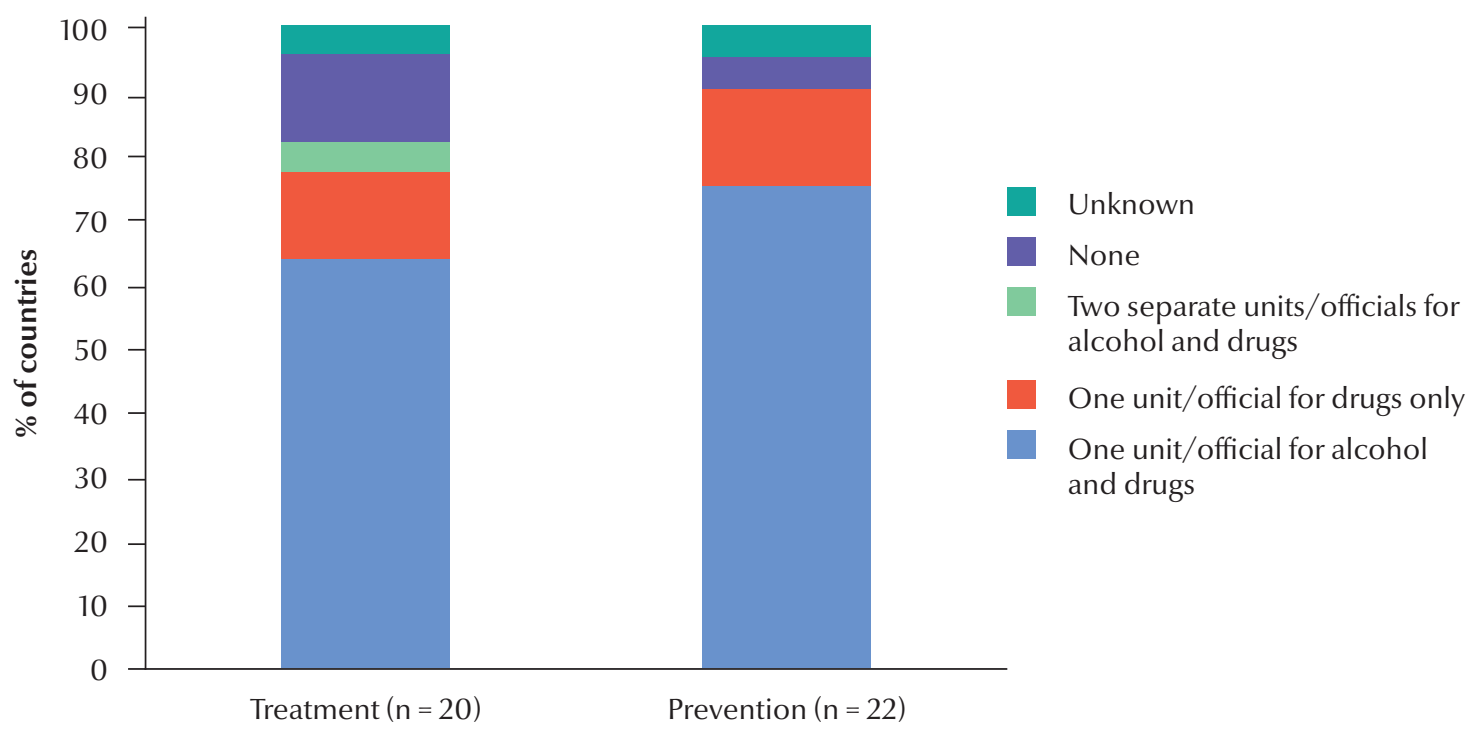

Figure 1 Government unit/focal person responsible for prevention and treatment of substance use disorders in countries of the Eastern Mediterranean Region

$71 \%)$ and under health promotion in just over a third $(5 / 14,36 \%)$.

The Atlas questionnaire asked about the ministry primarily responsible for prevention of substance use and treatment of substance use disorders. For the majority of countries (17/19,89\%), the ministry of health is responsible for prevention of substance use. The ministries of interior $(11 / 19,58 \%)$, social welfare $(7 / 19,37 \%)$ and education $(7 / 19$, $37 \%)$ were also reported to be responsible for prevention in some countries. Regarding treatment, again the ministry of health was the responsible ministry in most countries (19/20,95\%), followed by the ministry of interior $(6 / 19,30 \%)$.

\section{Financing}

Fourteen out of $21(67 \%)$ countries have a specific budget at the ministry of health for prevention of substance use, and 15/19 (79\%) countries report a specific budget at the ministry of health for the treatment of substance use disorders. Almost half of the countries have a budget line for prevention at the ministry of social welfare and a third at the ministry of criminal justice. On the other hand, 4 countries (Jordan, Lebanon, Somalia and Palestine) have no specific budget for prevention and 2 (Jordan and Palestine) have no specific budget for treatment.

Financing of treatment for substance use disorders can come from different sources, such as government, employers, households or through external financing such as nongovernmental organizations (NGOs). In most countries of the Region, the government is the main financing source for treatment, while 3-4 countries (depending on the treatment type) report that households are the main source of funding. The different treatment services provided are shown in Figure 2.

\section{Service organization and delivery}

\section{Treatment sectors and providers}

Treatment services for substance use disorders are provided by the public health sector in the majority of the countries (between 50\% and 77\% depending on the treatment service). The private health sector is reported to be the main sector for outpatient detoxification by just over a quarter of the countries (4/14, 28\%). One country (Sudan) reported that the criminal justice sector is the main sector providing treatment for substance use disorders and 2 countries (Pakistan and Egypt) reported that NGOs are the main providers of rehabilitation services.

Of the 22 countries, 7 (32\%) have specialized treatment facilities available in all different areas of the country (in major cities as well as in other urban and rural areas), 5 (23\%) have such facilities in the capital city only, 3 (14\%) have facilities in the capital city and other major cities and 1 (4\%) has only in rural areas. However, 6 countries (27\%) reported having no specialized treatment facilities for substance use disorders, whether integrated with other health care facilities or stand-alone:

In terms of the most important providers of treatment services, standalone specialized treatment services were reported to be the main providers in 8 countries (45\%), while 7 (39\%) reported mental health care facilities as the main providers.

\section{Legislative provisions for treatment}

The Atlas questionnaire asked about the existence of a law that protects the confidentiality of people in treatment for substance use disorders. Six countries (Iraq, Lebanon, Libya Pakistan, Sudan, 


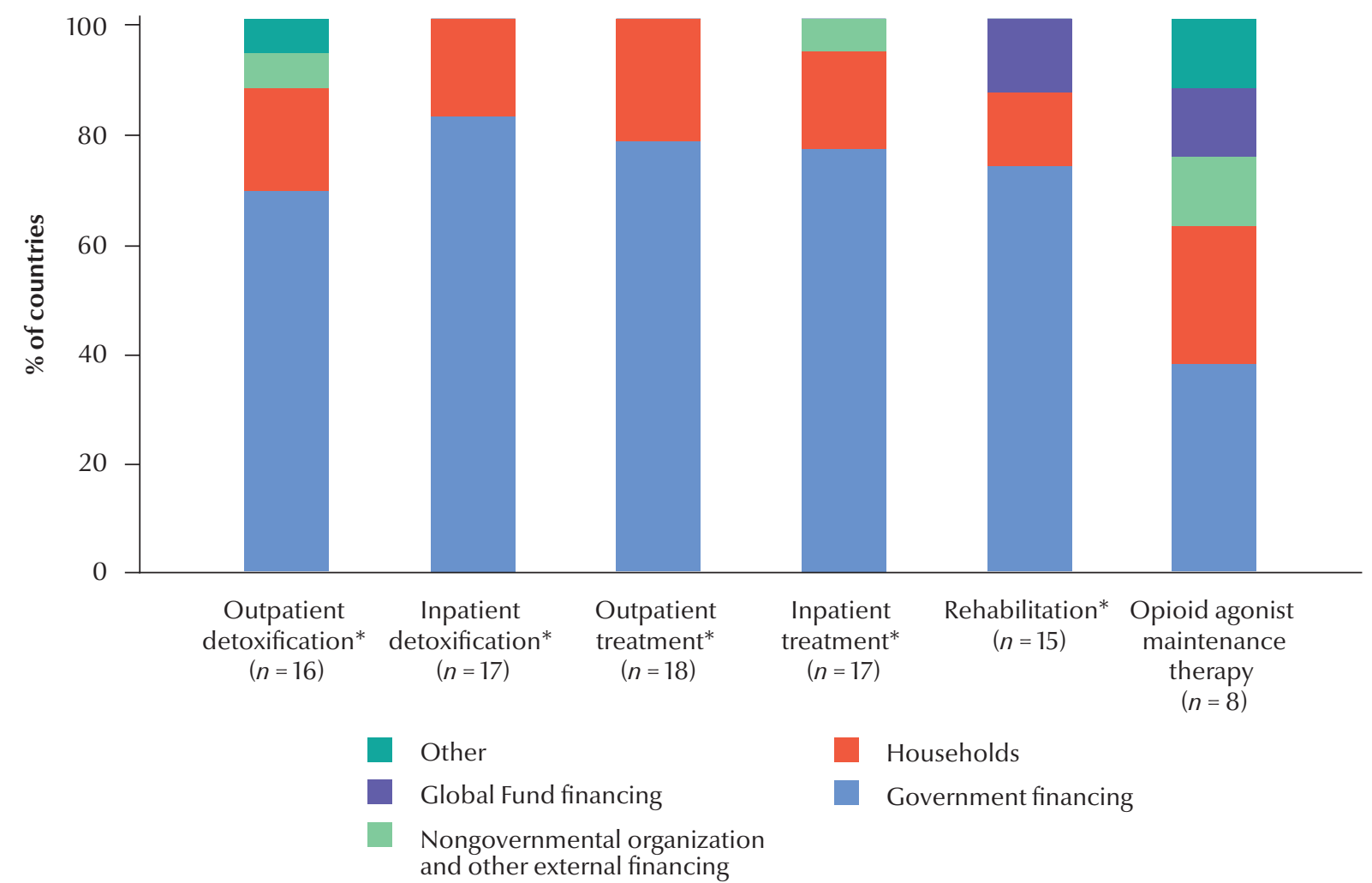

Figure 2 Main financing sources for treatment services for substance use disorders

Palestine) reported that they did not have such a law.

People in treatment for substance use disorders may have entered under their own volition or through formal or informal coercion. More than half of the countries have legislative or administrative provisions to offer voluntary treatment to people who come into contact with the criminal justice system, either as an alternative $(13 / 20,65 \%)$ or in addition $(11 / 18,61 \%)$ to criminal penalties. Mechanisms for compulsory treatment as an alternative or in addition to criminal penalties were reported less often than legislation for voluntary treatment: 9 out of 20 countries (45\%) reported having such mechanisms in place as an alternative to criminal penalties and 10 out of 19 countries (53\%) had compulsory treatment in addition to criminal sanctions.

\section{Main substance of concern}

The Atlas questionnaire asked about the main substances that resulted in entry into treatment. Opioids and cannabis were both reported by just over a quarter of the countries $(6 / 22,27 \%)$ as the main substances responsible for treatment entry. In addition, $5 / 22$ countries $(22 \%)$ reported them as the second most used substances. Four countries (Jordan, Qatar, Somalia and Sudan) reported alcohol as the main substance that led to treatment entry. Sedatives, anxiolytics and sleeping pills were reported as the third most used substances that led to treatment entry by $45 \%$ of the countries.

\section{Service coverage, capacity and utili- zation}

Treatment coverage is defined here as the proportion of the estimated number of people with drug dependence that are currently receiving treatment. It is a key indicator to assess service gaps. Most countries (13/22) could not provide information on treatment coverage as the data were not available. Out of the 9 countries that provided data, 4 (Bahrain, Islamic Republic of Iran, Saudi Arabia and the United Arab Emirates) reported treatment coverage of greater than $20 \%$. Regarding cannabis dependence, 3 countries (Bahrain, Oman and Saudi Arabia) reported treatment coverage of more than $20 \%$.

In addition to treatment coverage, countries were requested to provide information on the proportion of people who seek treatment for substance dependence who actually receive such treatment; this reflects access to treatment. Of the 11 countries who provided data for opioid dependence, 4 (Jordan, Kuwait, Oman and Qatar) reported that more than half of the people who sought treatment for opioid dependence received it. Of the 10 countries that provided data for cannabis dependence, 3 (Kuwait, Oman and Qatar) reported that more than half of those who sought treatment for cannabis dependence received treatment. 
Service availability can be assessed by the number of beds available for the treatment of substance use disorders, bearing in mind that not all treatment of substance use disorders requires hospitalization. Thirteen countries provided data on the number of beds. The median number of beds for treatment of substance use disorders in the Region was 0.73 per 100000 population (Table 1 ). It ranged from 0.05 (in Iraq) to 7.88 (in Kuwait) per 100000 population.

In terms of service utilization, the Atlas questionnaire asked about the annual number of treatment episodes for substance use disorders. In this context, treatment episodes refers to the total number of annual treatment admissions to inpatient and outpatient specialized treatment facilities, including the repeated treatment admissions for the same person during the year. Seven countries provided data on the number of outpatient treatment episodes in public sector specialized treatment facilities. The regional median was 32 annual treatment episodes per 100000 population, ranging from 2.4 (in Jordan) to 663 (in Bahrain) per 100000 population. For inpatient treatment episodes, the median was 9 annual treatment episodes per 100000 population with a low of 1.5 (in the United Arab Emirates) and a high of 57.5 (in Afghanistan).

\begin{tabular}{lc}
\hline $\begin{array}{l}\text { Table } 1 \text { Bed availability for inpatient treatment of substance use disorders in the } \\
\text { Eastern Mediterranean Region }\end{array}$ & $\begin{array}{c}\text { Median number of beds (per } \\
\mathbf{1 0 0} \mathbf{0 0 0} \text { population) for treatment } \\
\text { of substance use disorders }\end{array}$ \\
\hline Facility & 1.11 \\
Public general health care facilities $(n=2)$ & 0.17 \\
Public mental health care facilities $(n=3)$ & 0.92 \\
Private mental health care facilities $(n=1)$ & 0.80 \\
Public specialized health care facilities $(n=10)$ & 0.70 \\
Private specialized health care facilities $(n=2)$ & 1.33 \\
Other public health care facilities $(n=2)$ & 0.73 \\
Total $(n=13)$ &
\end{tabular}

Includes specialized health care facilities for substance use disorders only and does not include specialized mental health care facilities that offer specialized treatment for substance use disorders reported by just over half of the countries $(10 / 19,53 \%)$.

Prevention programmes exist in different settings in the Region. School-based prevention programmes for drug use are available in 14 of the 22 countries (64\%), with 3 reporting a high coverage. Fewer countries (50\%) reported community-based prevention programmes; 3 of them report a high coverage. Three countries (Lebanon, Somalia and Sudan) have no prevention programmes for drug use.

Substance use prevention programmes are available for different population groups; for young adults and prisoners in 14 out of 18 countries (78\%) and for people living with HIV/AIDS in 11 countries (61\%). We considered prevention programmes for people living with HIV/AIDS different from harm reduction programmes as they likely target people with HIV/ AIDS who do not use substances. Less than a quarter of the countries $(4 / 18)$ have specific drug use prevention programmes for people with mental disorders.

Screening and brief interventions for harmful and hazardous drug use in primary health care services and antenatal services are rare: $14 \%(3 / 21)$ of the countries reported that screening and brief interventions are implemented in $1-10 \%$ of primary health care services and antenatal services.

\section{Special programmes and services}

Harm reduction programmes are not widely available in the Region. A third of the countries (7/21) have needle/ syringe exchange programmes and $7 / 22$ have condom distribution programmes. Outreach services for injecting drug users are available in a quarter of the countries $(5 / 20), 3 / 20$ countries have drop-in services and 2/19 have naloxone distribution services.

Treatment programmes specifically allocated to women with drug use disorders are available in a third of the 
countries $(7 / 21)$; they are mainly located in the capital city or other major cities. Children and adolescents also represent a population group with specific needs; 4/19 (21\%) countries reported having special treatment programmes for children and adolescents with drug use disorders.

Housing and employment services for people with drug use disorders were reported by 3 countries (Islamic Republic of Iran, Saudi Arabia and the United Arab Emirates). Open access interventions such as telephone helplines, web-based or mobile phone based interventions are available in a small number of countries (7/20). Mutual support/self-help groups are available in 8/22 (36\%) countries and mostly located in the capital city and other major cities of the country.

\section{Workforce}

A relatively wide range of professionals provide treatment and care for the management of substance use disorders.
Psychiatrists were reported by all 22 countries in the Region, and psychologists by 20. Psychiatric nurses and social workers were reported by 14 and 16 countries respectively. Addiction specialists were less frequently reported (in 5 countries).

National standards of care were common for psychiatrists (in 16/18 countries, $88 \%$ ) and psychologists (in $11 / 18$ countries, $61 \%)$. Half of the countries $(9 / 18)$ have standards of care for social workers, but they were less frequently reported for other professional categories (Figure 3).

Education in prevention and treatment of substance use disorders such as short-cycle tertiary education (i.e. vocational, academic and professional education), and graduate and postgraduate degrees are available in less than half of the countries in the Region. When education programmes are available, short-cycle tertiary education in prevention of substance use was the most common and was reported by 9 of $22(40 \%)$ countries. A third of the countries $(7 / 21)$ have no training programmes for substance abuse. Similarly, 9 (41\%) countries have no specific postgraduate training programmes for professionals. The highest availability of postgraduate training programmes is for psychiatrists (11/22) (Figure 4). Continuing professional education for substance use prevention and treatment is available in 15/21 (70\%) countries, with programmes for psychiatrists being the most common (available in 14 countries).

\section{Information systems}

Data collection is a key element of a functioning health system and is the basis to inform relevant stakeholders. Only 8 of 20 (40\%) countries in the Region have a system that collects epidemiological data on substance abuse. These data incorporate results of regular epidemiological studies on the prevalence of substance use and substance use disorders, as well as patterns of

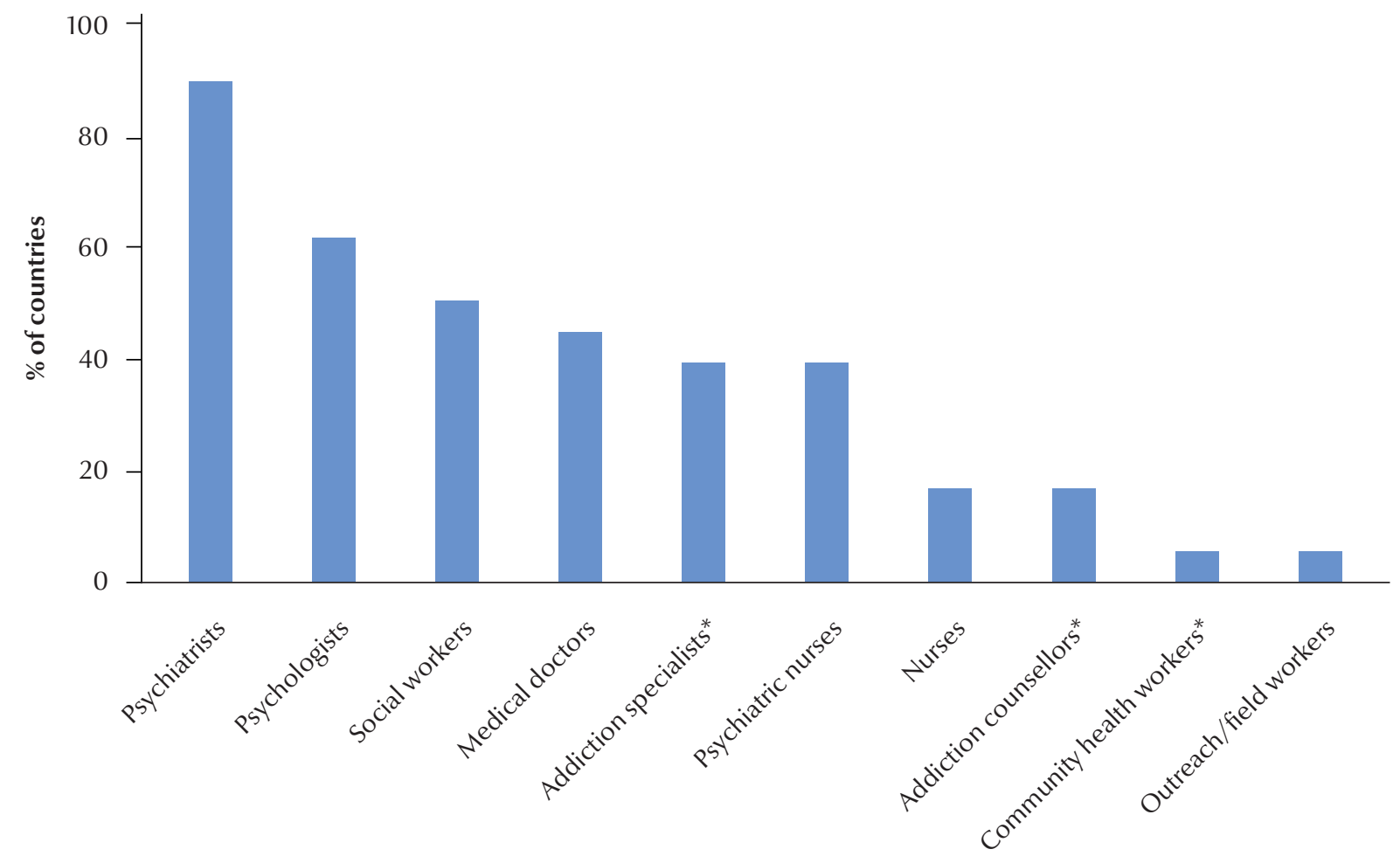

Figure 3 National standards of care for professionals providing treatment for substance use disorders $(\mathrm{N}=18)$ 


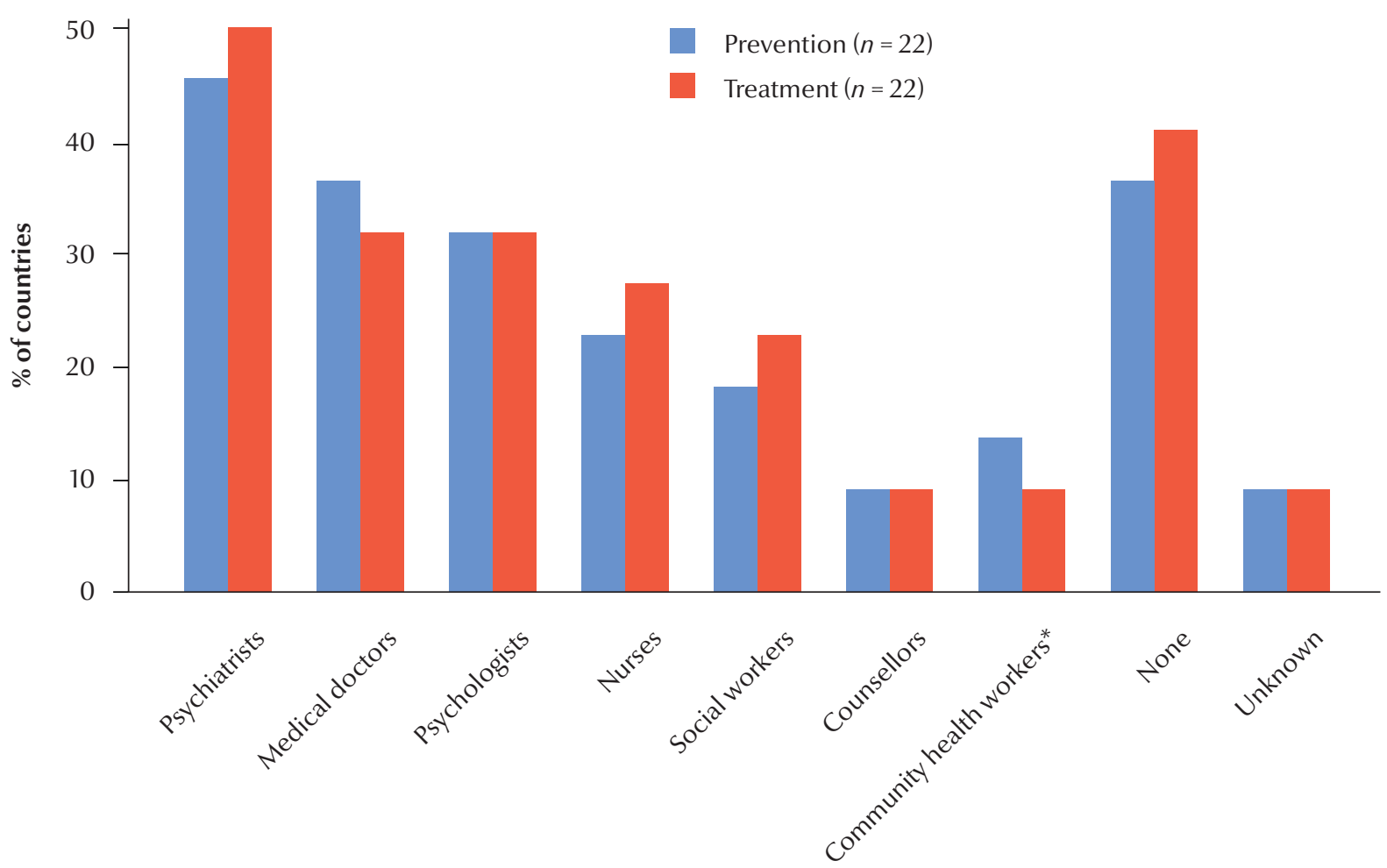

Figure 4 Availability of postgraduate training programmes in prevention and treatment of substance use disorders for different professionals

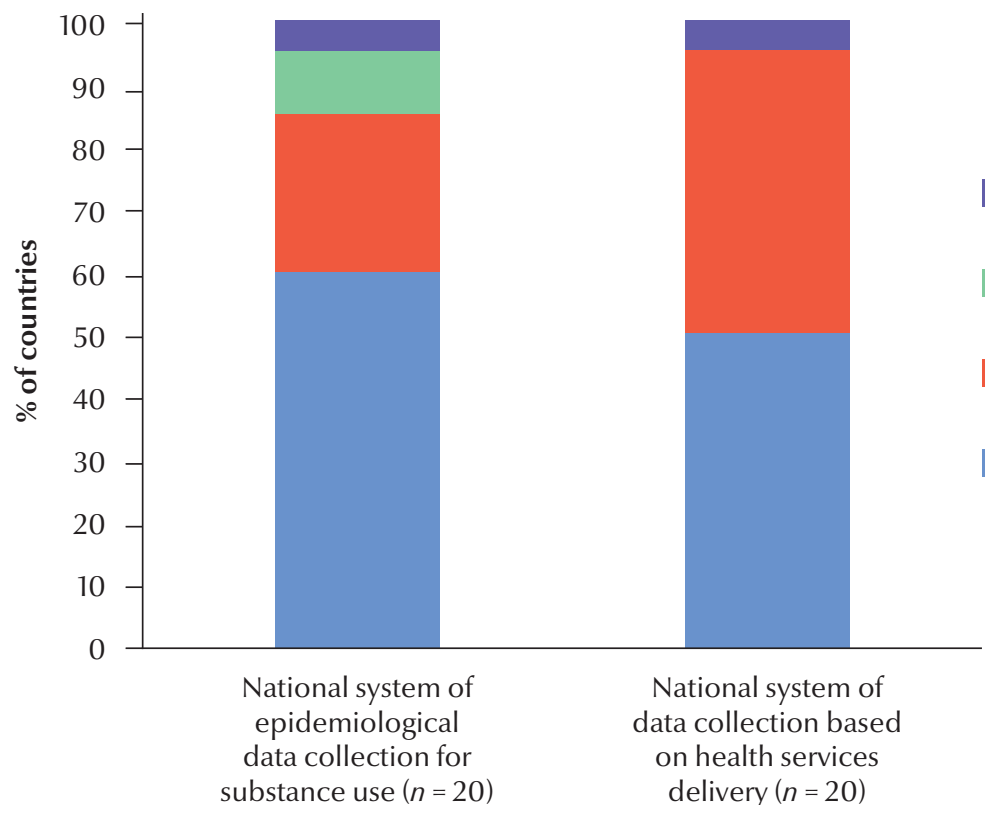

Two separate data collection systems for alcohol and drugs

One data collection system for drug use only

One data collection system for both alcohol and drug use

None

Figure 5 Existence of data collection systems for epidemiological data and data based on health services delivery for substance use and substance use disorders 


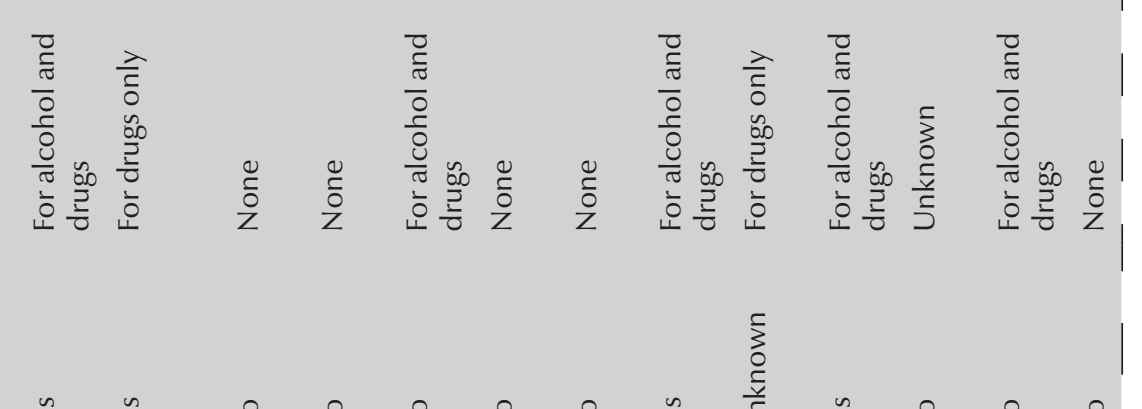

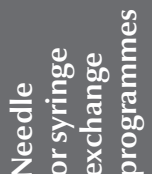

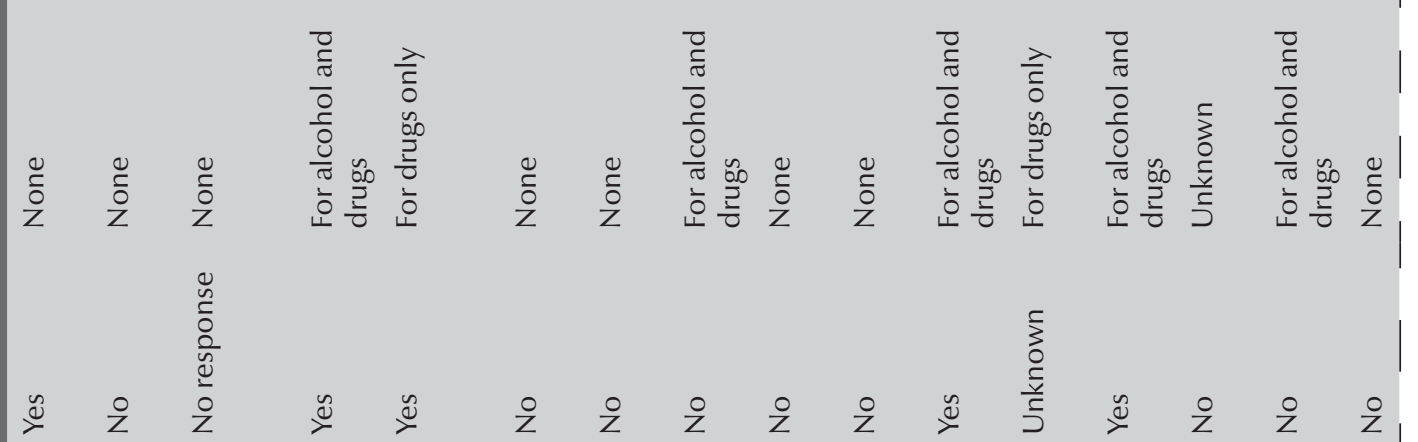

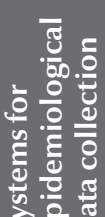

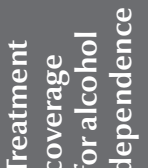

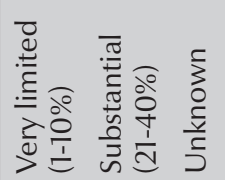

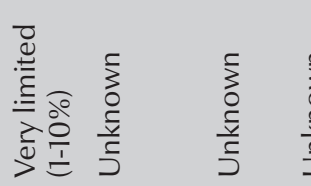

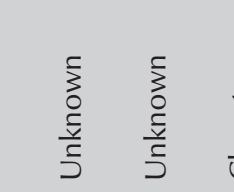

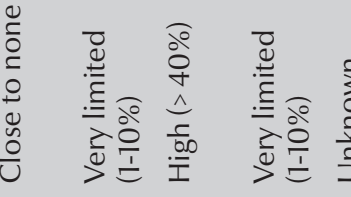

$-\quad 0$

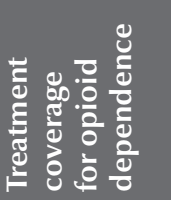

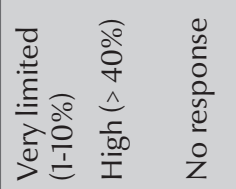

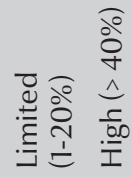

$\frac{5}{5}$
$\frac{5}{5}$
$\frac{5}{5}$

$\begin{array}{ll}\frac{5}{3} & \frac{5}{3} \\ 0 & 5 \\ \frac{5}{5} & \frac{5}{5} \\ \frac{5}{5} & 5\end{array}$

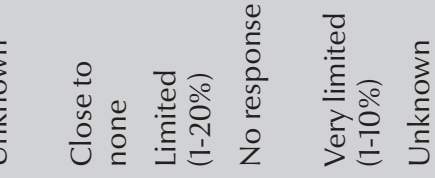

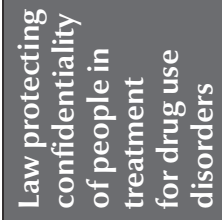

$\stackrel{\mho}{\check{0}}$

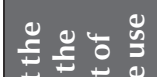

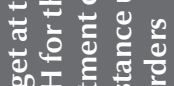

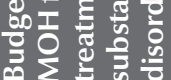

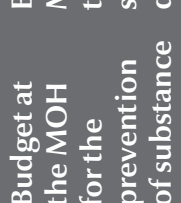

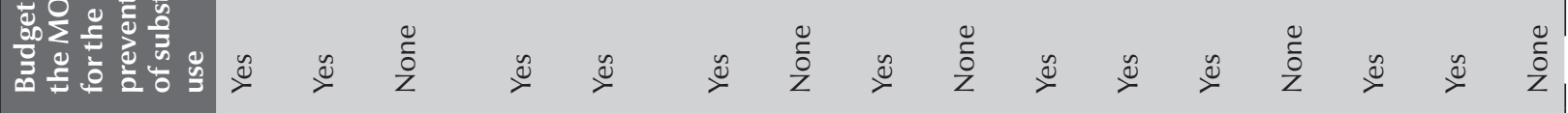

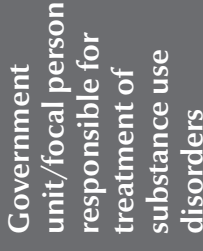

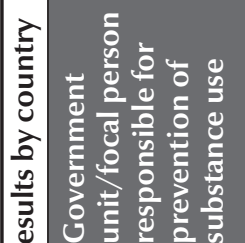

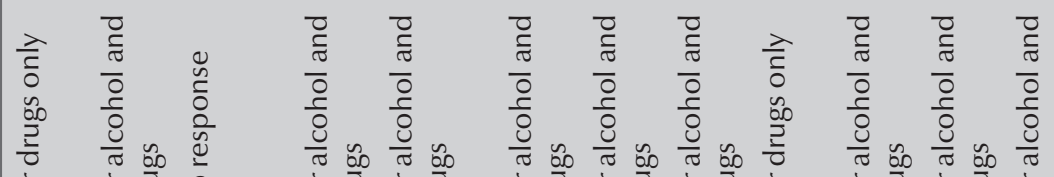

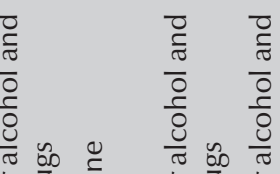

$\frac{\mathscr{D}}{\grave{0}}$

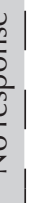

1

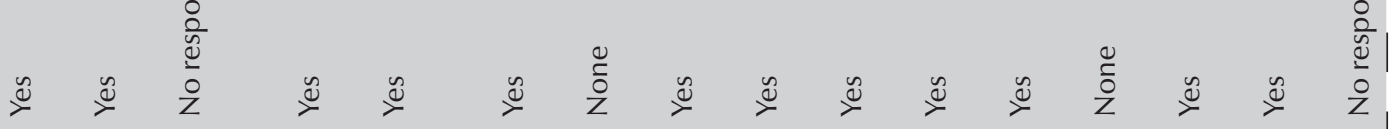

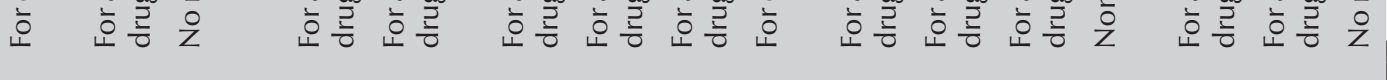




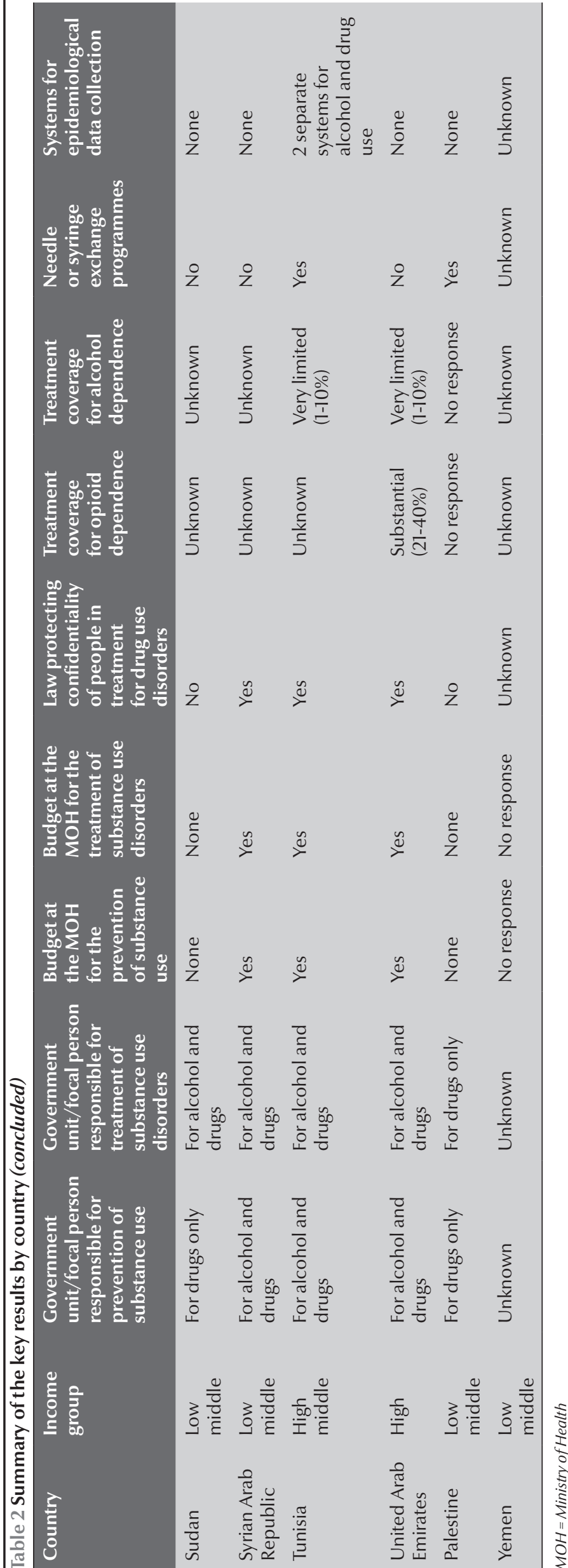

substance use. Half of the countries $(10 / 20)$ have a data collection system for health services delivery (Figure 5). Data on health services delivery typically include admission and discharge information as well the number of outpatient contacts. Half of the countries have reported data on substance use in the past 5 years.

Information on mortality and morbidity related to substance use can be collected by monitoring deaths related to substance use in forensic pathology. Just over a fifth of the countries (5/22) report such systems in forensic examinations or toxicology units. Table 2 gives a summary of the responses by the countries of the Region.

\section{Discussion}

This review presents the latest information on the resources available for the prevention and treatment of substance use disorders in the Eastern Mediterranean Region. All 22 of the countries of the Region submitted the Atlas questionnaire. Compared with the previous Atlas exercise, in which 14 countries participated, this increase may indicate a stronger political involvement in this public health issue. However, the lack of responses on some indicators should be kept in mind when looking at the results. The results show the paucity of information available as well as the lack of resources at the country level.

The areas covered in this review represent the 6 building blocks of a functioning health system. The leadership/governance and health information systems are the basis for the whole system. The financing and workforce components are at the core of the system, and the service delivery, including the availability of medications, represents the output of the health system (13). Our results show that more than $80 \%$ of the countries have a government unit or focal person responsible for the prevention and treatment of substance use disorders. However, only $40 \%$ have an epidemiological data collection system for substance use. The availability of data is essential to inform policy-makers and guide the development of treatment and prevention programmes. Resources should therefore be allocated to improve and develop the health information system in countries of the Region.

Regarding the financing aspect of the health system, the proportion of the countries (67\%) reporting having specific budgets allocated to the 
prevention of substance use is almost the same as the global average (69\%). A slightly higher proportion of countries in the Region had budgets allocated for the treatment of substance use disorders compared with the global average (79\% and $72 \%$ respectively). Budgets are structured differently in the Region as budgets for substance use are integrated with mental health and not with health promotion or noncommunicable diseases as is the case in other Regions.

The availability of human resources and training in the Region is similar to global averages. A lack of training for professionals is commonly reported, particularly in lower income countries.

Although there is a lack of recent epidemiological data on the extent of drug use, nonetheless the prevalence of drug use in the Region appears to be comparable with the global estimates, but the overall availability of services is low. Medications for maintenance treatment of opioid dependence are available in only half of the countries despite their benefits and the affordability of methadone, for instance. Open access interventions such as telephone help-lines or web-based interventions are half the global average. Needle/ syringe exchange programmes are one of the harm reduction interventions recommended by the WHO (14) to reduce the overall burden attributable to drugs. However, such interventions are available in only a third of the countries of the Region. Similarly, naloxone distribution programmes were reported by only 2 countries; recent WHO guidelines recommend the use of naloxone in emergency management of suspected opioid overdose (15). Compared with other WHO regions, the Eastern Mediterranean Region has the lowest proportion of countries implementing screening and brief intervention programmes. Globally, 23\% of countries report that screening and brief interventions are implemented in
1-10\% of primary health care services and antenatal services, while $14 \%$ of countries in the Region reported these interventions were implemented. Such programmes have been shown to be effective in addressing harmful alcohol use in primary health care settings (16).

The economic context varies across the countries in the Region. According to the World Bank classification, 5 are high income countries, 6 are high-middle income, 8 as low-middle income and 2 as low income countries (Table 2). Some indicators reported in this review seem to be related to the income group. For instance, all high income countries reported having a government unit/focal person as well as a budget line at the ministry of health. Among the high middle income countries, 2 reported having no budget line at the ministry of health for prevention of substance use (Jordan and Lebanon). Treatment coverage also seems to correlate with the income category. Countries that reported treatment coverage for opioid dependence of more than $20 \%$ were all high income countries, with the exception of the Islamic Republic of Iran. Harm reduction measures, such as the availability of needle/syringe exchange programmes, however, do not appear to be related to income group. Apart from the Islamic Republic of Iran and Tunisia, which are high middle income countries, needle/syringe exchange programmes are available in low and low middle income settings (Afghanistan, Egypt, Morocco, Pakistan and Palestine).

The data presented in this review are based on the Atlas project and were reported by the focal points at the ministry of health or other responsible ministry. Focal points were strongly encouraged to consult with experts in the country but external data verification was not part of the process, and this should be borne in mind when considering the findings. As already mentioned, the lack of information reported for some indicators makes it difficult to assess the treatment gaps and properly inform decisions. A few components that are essential for a comprehensive treatment package for substance use disorders, namely structured psychosocial interventions, were not surveyed in the Atlas and therefore the relevant data could not be presented in the current review.

\section{Conclusion}

The current estimates show that the burden attributable to drug use disorders in the Region is high, which highlights the importance of considering substance use and substance use disorders in health policy. Health system resources for the prevention and treatment of substance use disorders vary across countries, but remain overall insufficient to provide adequate care and treatment for people with these disorders.

Social supports, such as government benefits, housing or employment services, are rarely available for people with substance use disorders. Interventions that have proved efficient, such as pharmacotherapy or screening and brief interventions, are only available in a small number of the countries of the Region. Such services should be scaled up to be available in all parts of the country and not limited to the major cities. Information systems should be strengthened and data collected in a systematic way in order to provide the information needed for planning services and resources. More importantly, to successfully implement the Sustainable Development Goals, countries are urged to strengthen the prevention and treatment of substance use disorders, particularly by increasing the coverage of treatment interventions for substance use disorders.

Funding: None.

Competing interests: None declared. 


\section{References}

1. World Health Oganization. Neuroscience of psychoactive substance use and dependence. Geneva: World Health Organization; 2004.

2. World Health Organization. Global status report on alcohol and health - 2014 ed. Geneva: World Health Organization; 2014.

3. World drug report 2016. Vienna: United Nations Office on Drugs and Crime; 2016.

4. World Health Organization. Atlas: substance use in the Eastern Mediterranean Region 2015. Cairo: WHO Regional Office for the Eastern Mediterranean; 2015.

5. Degenhardt L, Whiteford HA, Ferrari AJ, Baxter AJ, Charlson FJ, Hall WD, et al. Global burden of disease attributable to illicit drug use and dependence: findings from the Global Burden of Disease Study 2010. Lancet. 2013;382(9904):1564-74.

6. Babor T, Stenius K, Romelsjo A. Alcohol and drug treatment systems in public health perspective: mediators and moderators of population effects. Int J Methods Psychiatr Res. 2008;17 Suppl 1:S50-9.

7. World Health Organization. Everybody's business. Strengthening health systems to improve health outcomes: WHO's framework for action. Geneva: World Health Organization; 2007.

8. World Health Organization. Atlas on substance use 2010: resources for the prevention and treatment of substance use disorders. Geneva: World Health Organization; 2010.
9. World Health Organization. Atlas: substance use in the Eastern Mediterranean Region 2012. Cairo: World Health Organization Regional Office for the Eastern Mediterranean; 2013.

10. World Health Organization. Guidelines for the psychosocially assisted pharmacological treatment of opioid dependence. Geneva: World Health Organization; 2009.

11. United Nations Office on Drugs and Crime (UNODC). Political Declaration and Plan of Action on International Cooperation towards an Integrated and Balanced Strategy to Counter the World Drug Problem. Vienna: UNODC; 2009.

12. World Health Organization. Global strategy to reduce the harmful use of alcohol. Geneva: World Health Organization; 2010

13. World Health Organization. Monitoring the building blocks of health systems: a handbook of indicators and their measurement strategies. Geneva: World Health Organization; 2010.

14. World Health Organization. Consolidated guidelines on HIV prevention, diagnosis, treatment and care for key populations. Geneva: World Health Organization; 2014.

15. World Health Organization. Community management of opioid overdose. Geneva: World Health Organization; 2014.

16. O'Donnell A, Anderson P, Newbury-Birch D, Schulte B, Schmidt C, Reimer J, et al. The impact of brief alcohol interventions in primary healthcare: A systematic review of reviews. Alcohol Alcohol. 2014;49(1):66-78. 